\title{
CASA LBT 1961: ARTE CONCRETO CONSTRUIDO
}

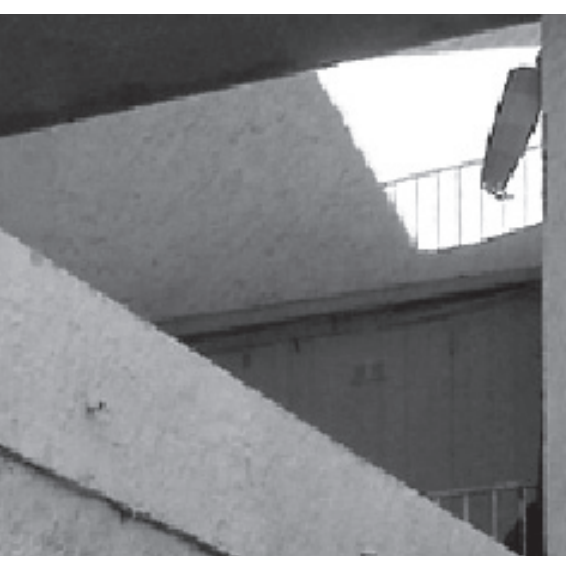

\author{
Héctor García-Diego Villarías y María Villanueva Fernández
}

LBT House 1961: Built Concrete Art

Boletín Académico. Revista de investigación y arquitectura contemporánea

Escuela Técnica Superior de Arquitectura. Universidade da Coruña

elSSN 2173-6723

www.boletinacademico.com

Número 5 (2015)

Páginas 19-26

Fecha de recepción 02.11.2014

Fecha de aceptación 08.01.2015

https://doi.org/10.17979/bac.2015.5.0.1035

\section{Resumen}

El artículo explora la operatividad de los principios gráficos del Arte Concreto en la aplicación a un proyecto real de arquitectura. Para ello, se utiliza el ejemplo de la casa de Lanfranco Bombelli proyectada por él mismo y situada en Cadaqués en 1961. La obra da muestra de un ensayo cabal de arquitectura doméstica que se nutre de algunas nociones gráficas precisas que se desprenden de su filia personal hacia esta corriente artística. Se trata ésta de una manera de afrontar la tarea artística que presenta ciertas similitudes con la manera de afrontar el proyecto de arquitectura. Y de ahí que la Casa LBT presente experimentalismo plástico palpable, que sin embargo se imbrica con una sofisticada relectura de los lenguajes que es sensible con la tradición local.

\begin{abstract}
The article explores the operability of the graphic principles of the Concrete Art in its application to a real architecture project. To achieve this goal, the text uses the example of Lanfranco Bombelli's own house, designed by him in 1961 and located in Cadaqués. The work demonstrates how domestic architecture can draws on notions of graphics. Actually, Concrete Art presents certain similarities in way of dealing with the project of architecture. And that is why the plastic experimentalism of the LBT house is palpable, yet overlaps with a sophisticated reading of the local tradition language.
\end{abstract}

\section{Palabras clave}

Arte concreto, Bombelli, Cadaqués, Casa

\section{Keywords}

Concrete Art, Bombelli, Cadaqués, Housing 
$\mathrm{L}, \mathrm{b}$ y $\mathrm{t}^{1}$. Tres iniciales dan nombre a la casa que se presenta a continuación, a la vivienda que Lanfranco Bombelli decidió construir en Cadaqués en el año 1961 para vivir de manera independiente respecto a su amigo y socio Peter Harnden (Fig. 01-03). L, b, y t son sólo tres letras que vienen a representar a un tiempo la rúbrica personal del que diseña la casa y del que espera vivir en ella: Lanfranco Bombelli Tiravanti.

Han pasado dos años desde la construcción de Villa Gloria y Bombelli, habida cuenta de las evidentes incomodidades que suponía compartirla con una familia tan numerosa como la de Harnden ${ }^{2}$, decide abandonar la casa y proyectarse una propia. Las estancias en la Costa Brava cada vez eran más habituales y más prolongadas, y la necesidad de disfrutar de un espacio emancipado para la familia Bombelli era cada vez más acuciante.

La Casa LBT es uno de los ejemplos de arquitectura más conspicuos de la pareja Peter Harnden y Lanfranco Bombelli en Cadaqués ${ }^{3}$. En ella se desarrollan los argumentos de los arquitectos presentes en otros tantos proyectos domésticos en el municipio. Así, la casa construida para el propio Lanfranco ${ }^{4}$, pone en práctica los mismos principios empleados por la pareja de arquitectos para proyectos similares en el mismo lugar ${ }^{5}$, como son la inserción en la singular trama urbana de Cadaqués, el encuentro de la nueva construcción con el paisaje natural o la incorporación de algunos de los valores constructivos y estilísticos locales.

La relación con el entorno se realiza por vía tanto de una acentuada simplificación formal, como por la de la integración del nuevo elemento en la constreñida trama urbana de Cadaqués. Concretamente, la Casa LBT se encuentra encajada entre dos viviendas a los lados, y delimitada por sendas calles en desnivel en las partes delantera y trasera ${ }^{6}$. Una importante diferencia de cota entre las distintas vías que llega a alcanzar las dos plantas de altura en total. Estas características han sido destacadas precisamente por los arquitectos gerundenses Carme Tena y Ferrán Lorenzo: «La Casa de Port Doguer se puede considerar una transcripción a escala de todo lo expuesto en el conjunto del pueblo, en lo que a vivienda unifamiliar se refiere. Está encajada entre dos calles paralelas desniveladas la altura de dos plantas; la roca que surge, como en tantos lugares de Cadaqués, en la parte posterior de la planta baja, irrumpe descaradamente en el interior de la vivienda como expresión máxima de la fuerza del lugar» ${ }^{7}$.
La casa se posiciona entre las construcciones previas y asume desde el primer momento el deseo de mirar sobre el conglomerado urbano de Cadaqués. Con este propósito, el programa se estira verticalmente, tratando de situar las estancias de mayor disfrute $-\mathrm{y}$ con mayor trascendencia para el mayor número de habitantes - en las posiciones más elevadas.

La vivienda se divide desde el primer momento en dos partes: Bombelli plantea un volumen sencillo y bastante hermético en la parte inferior, que a su vez sirve de podio sobre el que situar otra pieza bien distinta que corresponde al gran estar. Una operación que cuenta con algunas similitudes respecto a la llevada a cabo en Villa Gloria tiempo atrás, y que por tanto comparte sus mismas ventajas de inserción en el paisaje urbano al situar la zona más permeable - y por tanto significativamente transgresora con la apariencia del conjunto tradicional de Cadaqués - en una posición no divisable desde el exterior. De nuevo, una resolución del programa atractiva, pero que no resultaría agresiva al contexto del pueblo de pescadores y artistas.

No obstante, a pesar de este supuesto bipartidismo formal de partida, la casa de Lanfranco Bombelli presenta una aparente unidad compositiva que la diferencia sutilmente del Villa Gloria. La depuración de líneas que se percibe desde el primer momento de la contemplación del edificio, se lleva a todas las partes de la casa por igual, dando lugar al oscurecimiento de esta división del programa en su apariencia exterior. De este modo, Bombelli ofrece una arquitectura que, aunque en sintonía con la arquitectura anónima que le circunda, manifiesta abiertamente que se trata de un tipo de arquitectura de autor. Bombelli ofrece un tipo de arquitectura que se manifiesta abiertamente como impregnada de un trascendente sentido plástico. Una composición que, se intuye, podría captar su inspiración en los postulados compositivos del Arte Concreto al que tan entusiásticamente se sumó en el pasado.

Y es que el interés por esta corriente artística por parte del arquitecto — de la que también fue partícipe en varias ocasiones a través de su trabajo como artista y galerista ${ }^{8}$ - , le influiría notablemente en la manera de enfrentarse al problema creativo. De hecho, al ser preguntado por el origen de su casa en Cadaqués, no duda en afirmar esta evidente influencia: «Seguro que hay una influencia de mi origen italiano (...) algo que he absorbido inconscientemente, pero creo que hay también una influencia del Arte Concreto, de la composición geométrica, de las proporciones, del orden que existe en el Arte Concreto»?. 

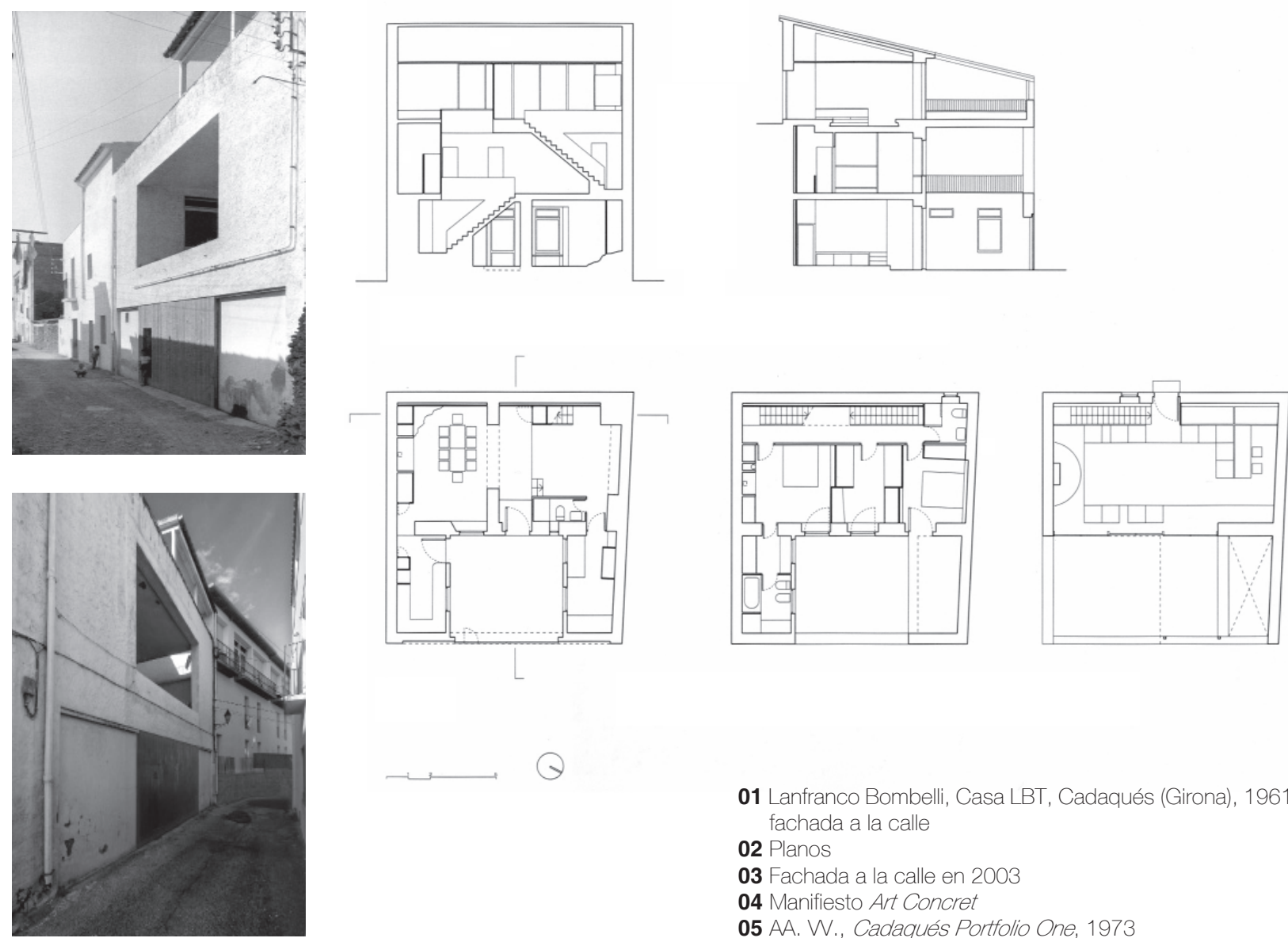

01 Lanfranco Bombelli, Casa LBT, Cadaqués (Girona), 1961; fachada a la calle

02 Planos

03 Fachada a la calle en 2003

04 Manifiesto Art Concret

05 AA. W., Cadaqués Portfolio One, 1973

\section{ART CONCRET}

GROUPE ET REVUE FONDÉS EN 1930 A PARIS

PREMIERE ANNEE-NUMERO DINTRODUCTION-AVRIL MIL NEUF CENT TRENTE

BASE DE LA PEINTURE CONCRÈTE

Nous disons

$1^{\circ}$ L'art est universel.

$2^{\circ} L^{\prime}$ 'euvre d'art doit être entièrement conçue et formée par l'esprit avant son exécution. Elle ne doit rien recevoir des domnées formelles de la nature, ni de la sensualité, ni de la sentimentalité.

Nous voulons exclure le lyrisme, le dramatisme, le symbolisme, etc.

$3^{0}$ Le tableau doit être entièrement construit avec des éléments purement plastiques, c'est-à-dire plans et couleurs. Un élément pictural n'a pas d'autre signification que "lui-même" en conséquence le tableau n'a pas d'autre signification que "lui-mêmen.

$4^{\circ}$ La construction du tableau, aussi bien que ses éléments, doit être simple et contrôlable visuellement.

$5^{\circ}$ La technique doit être mécanique c'est-à-dire exacte, anti-impressionniste.

$6^{\circ}$ Effort pour la clarté absolue.

Carlsund, Doesbourg, Hélion, Tutundjian, Wantz.
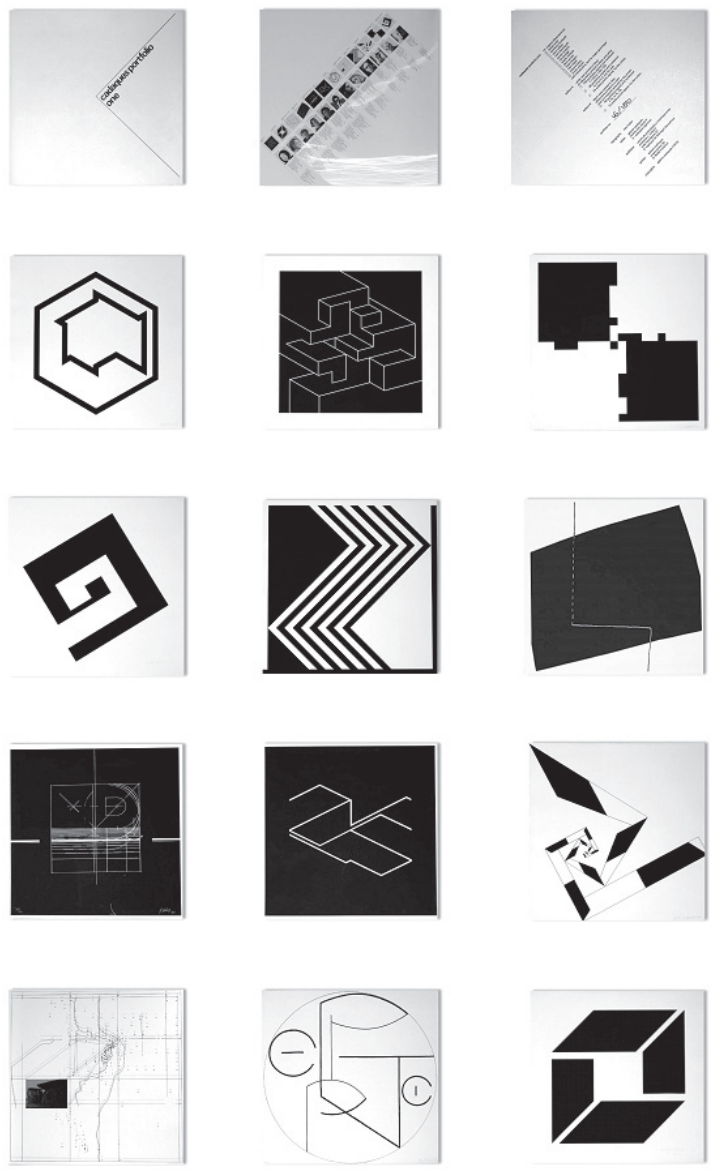
No obstante, no es el propósito de esta investigación evaluar las siempre complejas implicaciones que se producen al yuxtaponer arte y arquitectura. La relación entre arte y arquitectura ha sido una constante más que discutida a lo largo de la historia y para la que se han alcanzado dispares conclusiones. Sin embargo, en este caso se considera que la relación específica de Lanfranco con el arte sí presenta una influencia cierta, debido principalmente a las características particulares que representa esta corriente artística. De hecho, los postulados defendidos por el Arte Concreto se insertaban en una línea de trabajo especialmente propicia para poder ser puestos en marcha también en el ejercicio de la arquitectura ${ }^{10}$.

Una de las características fundamentales de los artistas concretos sería la de vincular la idea de arte a la de proyecto. Según esto, se otorga cierta operatividad al arte. En cierto modo, se aspira a un arte útil que puede ser aplicado a otras disciplinas como el diseño de mobiliario, la tipografía o la arquitectura. Algo presente en algunos de los artistas suizos de mayor reconocimiento seguidores de esta tendencia artística en aquella época, como Max Bill o Jean $\operatorname{Arp}^{11}$. Éste último así lo describía: «No queremos copiar la naturaleza. No queremos reproducir, queremos producir. Queremos producir como una planta produce la fruta y no la reproduce. Queremos producir directamente, no de segunda mano. Como no hay el menor rastro de la abstracción en este arte lo llamaremos arte concreto» ${ }^{12}$.

O, en palabras de Max Bill, amigo personal de Bombelli y sin duda una de las personas que más pudo influirle en este sentido: «La aparición de este nuevo arte es el resultado de una visión que se mueve en un terreno accesible al pensamiento y que, en un sentido, ofrece no pocas seguridades, pero que, en otro, es una invitación a lo desconocido, a lo indefinible; es decir, que se mueve en una región límite que permite abrir nuevos campos de visión y hacerlos perceptibles sensorialmente» ${ }^{13}$.

No obstante, debe aclararse que el nacimiento de esta tendencia artística se remonta al año 1930 con el manifiesto promovido por Theo van Doesburg en el primer y único número de la revista Art Concrett $^{14}$ (Fig. 04). Fue la primera vez que se enunció dicho término, si bien es cierto que la muerte del pintor holandés al año siguiente truncó en buena medida su desarrollo en los círculos artísticos de la capital francesa. Sin embargo, este movimiento plástico ${ }^{15}$ fue desarrollado con mayor profundidad entre grupos artísticos de la escena suiza — como el grupo Allianz—, lo que propi- ció la familiarización de Bombelli con esta manera de acercarse al arte durante su estancia en el país alpino en los años cuarenta.

De modo que la Casa LBT se materializa conforme a una composición concreta. Una elegante y sencilla construcción plástica que se compone, en fachada, nada más que de un gran hueco superior y una réplica inferior que se esconde tras un liviano plano de madera. Un generoso portón se desliza en el lienzo de la composición como si se tratara de una especie de velo que se interpone entre exterior e interior. Un plano abstracto que se instala, por un lado, en el mundo de la abstracción pura del arte concreto, pero que por otro, anuncia la posibilidad de desdibujar estos límites en su posible oscilación y dar acceso a una casa real.

Buena muestra de esta reminiscencia concreta es, precisamente, la obra gráfica que Lanfranco presentó junto a algunos de sus amigos y artistas para honrar la memoria de Peter Harnden en la carpeta Cadaqués Portfolio One ${ }^{16}$ (Fig. 05). Su propio trabajo cubo cinque es un ejemplo evidente de su predilección por esta manera de entender el arte. Pero además, da constancia de un ejemplo que revela en cierto modo una manera plástica de acercarse al ejercicio compositivo que parece compartir algunos rasgos con la Casa LBT (Fig. 06-07).

En la lámina de 50 x $50 \mathrm{~cm}$., Bombelli realiza una composición en blanco y negro perfectamente afín al arte concreto. El hecho de prescindir del color viene a ceder todo el protagonismo a la forma, que de este modo predomina sobre cualquier otra característica del trabajo. Un aspecto absolutamente exportable al campo de la arquitectura donde es habitual la preeminencia de la forma sobre otras variables. Y también en el caso de su propia casa.

Además, la composición de cubo cinque aspira claramente a una acentuada centralidad formal. Es centrífuga y centrípeta al tiempo, blanca y negra a la vez. Los cuatro planos en tinta plana se reúnen en torno a lo que parece ser un cuadrado central que queda definido por la presión de estos elementos a su alrededor. Se trata de un ejercicio análogo en buena medida a aquellos propios de la psicología de la gestalt ${ }^{17}$, quizá más concretamente de aquellos ejemplos que se conocen habitualmente como percepción multiestable, en donde su establece un juego perceptivo entre las partes y el todo ${ }^{18}$.

Pero además resulta reveladora la tensión que se establece entre la bidimensionalidad de la lámina —con pares de elementos iguales, líneas diagonales alternamente 


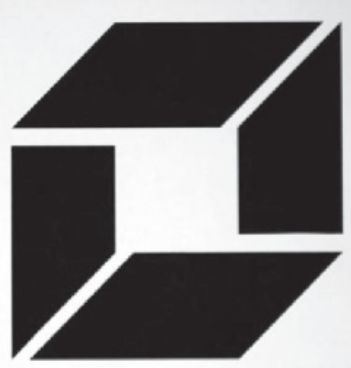

06 Lanfranco Bombelli, Cubo Cinque 07 Casa LBT; escorzo en 2003

08 La terraza vista desde el interior 09 Estancia
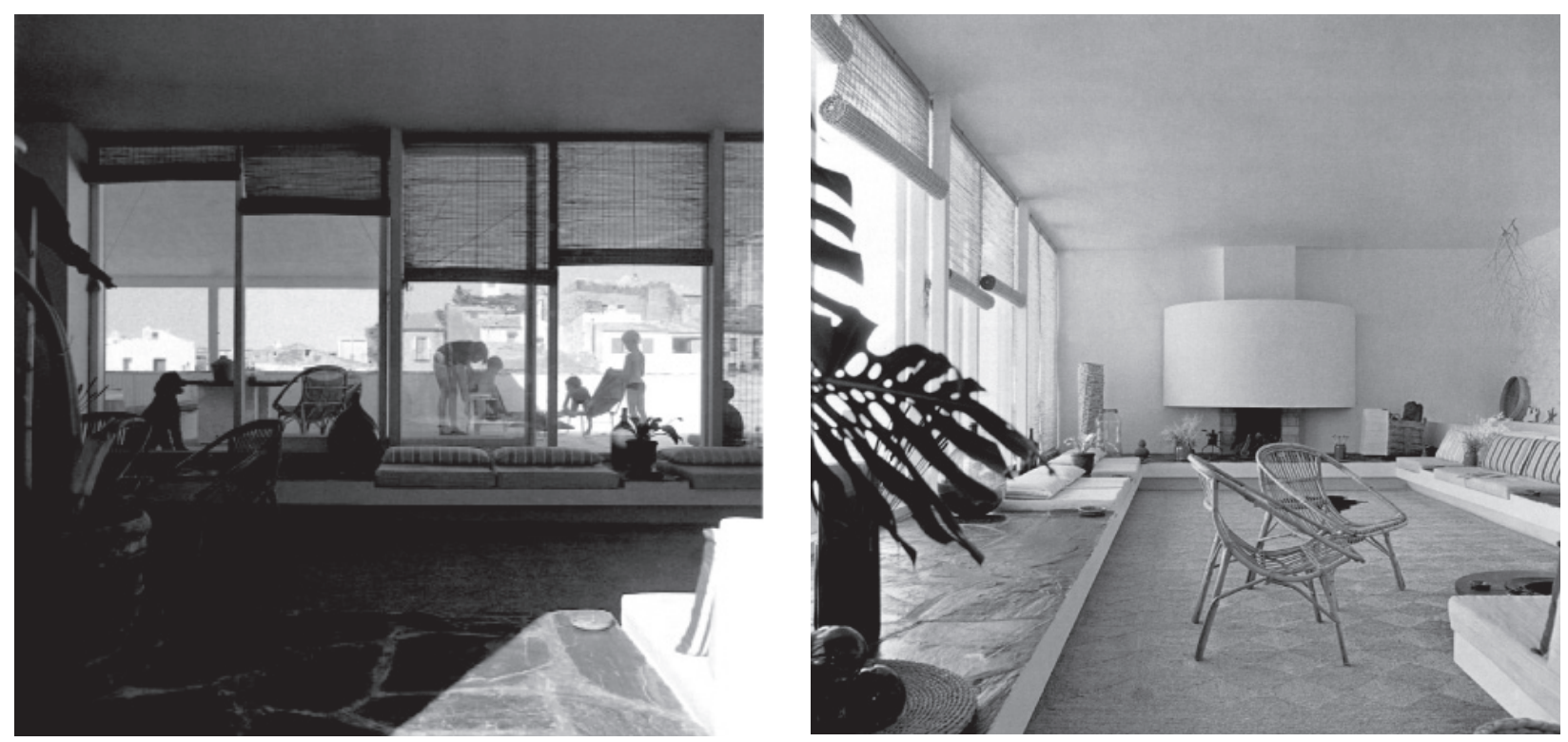
coincidentes_- y su tridimensionalidad — con planos negros que sugieren profundidad-, coincidencia ambigua de unas posibles aristas que evocan una determinada volumetría, etc. Esta ambigüedad espacial — sin duda la característica más destacable de la obra- presenta a las claras la especial línea de trabajo liderada por Bombelli.

Si en las dos dimensiones la lámina se limita a ordenar unas formas simples que invitan a hacer una lectura simultánea entre positivo y negativo en la percepción tridimensional de la obra, la invitación es incluso más poliédrica y ambigua. Mientras que los dos planos paralelos superior e inferior parecen situarse en perpendicular al plano del observador, los otros dos planos colocados a ambos lados pueden ser interpretados en dos direcciones transversales diferentes: bien en perpendicular o en paralelo al plano del cuadro. El resultado es la insinuación de un espacio cúbico virtual que ofrece visiones simultáneamente diferentes.

Pero además, y en esta misma línea narrativa, la composición de Bombelli es un lienzo plano, compuesto de dos grandes rectángulos perfectamente modulados. Y es un volumen tridimensional, sugerido desde la composición bidimensional pero decididamente ambiguo, en tanto en cuanto se proyecta en diferentes direcciones espaciales por el interior de la vivienda. Como cubo cinque, el vacío que serpentea por el interior de la casa de Bombelli nace del hueco frontal predominante, para a continuación desplazarse en diferentes direcciones para así hacer ambigua su percepción y enriquecer todas las posibles lecturas de la composición.

Es por esto que el argumento principal del edificio, su vacío, zigzaguea por el interior del volumen. Pero no sólo se trata de composición pura; es arquitectura. Se detiene en pequeños espacios y huecos que van dotando de luz las diferentes dependencias de la vivienda para estallar en una explosión luminosa en la planta superior. Todo aquello que en cubo cinque - en el plano meramente compositivo- eran líneas, planos y volúmenes, ahora se pone al servicio de la mente del arquitecto para crear los diferentes espacios, aunque partiendo, eso sí, desde la unidad que se le presupone al rotundo argumento plástico que emplea Bombelli.

Además, en línea con esta posible interpretación plástica de la Casa LBT, cabe destacar la importancia que para Bombelli tenía el hecho de proveer a su casa de un discurso compositivo ambicioso y acorde a sus convicciones. En este sentido resulta más que comprensible la versión final del proyecto, ya que en algunos de los estudios preliminares el hueco central de la fachada, se desplazaba a uno de los lados. De este modo, en las versiones más tempranas se optaba por una ordenación en forma de «L» — a diferencia del esquema final presentado aquí en «U» (Fig. 02) — lo que facilitaba en gran medida la ordenación en planta de la casa ${ }^{19}$. Sin embargo, Bombelli decide finalmente vaciar la volumetría desde el centro, dando prioridad a la composición plástica de la casa sobre la optimización de la distribución programática.

Con todo, el vaciado de la casa dota a toda ella de un significado de conjunto al tiempo que singulariza cada una de las partes. La operación general procedente de un gesto calculado de la mano del artista, desemboca en la definición precisa y singular de cada una de las partes con arreglo a un mismo proyecto. Y si este fenómeno se produce en la organización de la vivienda, lo mismo ocurre en su dimensión constructiva.

Así, en este recorrido vertical, el vacío supone una excusa perfecta para que el arquitecto despliegue una gran cantidad de recursos propios del profesional experimentado en el oficio. Bombelli emplea cualquier accidente en torno al patio y la ascensión por las escaleras para dar lugar a espacios con una función ambigua y, quizá por eso, espacialmente sugerentes. De esta manera, la escalera adopta una dimensión que trasciende a la mera utilidad que se le presupone, y además subraya una vez más la composición centralizada el patio: «El dominio de los diferentes niveles y repertorios de recursos, con rellanos para enfatizar y conseguir la continuidad interior-exterior, rellanosasiento y rellanos-peldaño, están utilizados con una notable facilidad y sencillez en esta casa. Así mismo, la escalera como elemento vertebrador se desarrolla en cada planta desde los extremos hacia el centro para reforzar la centralidad del patio» ${ }^{20}$.

Bombelli utiliza los elementos presentes en Cadaqués, pero los ordena conforme a un discurso de clara modernidad. De este modo, el lenguaje arquitectónico se enriquece al yuxtaponer elementos de mayor y menor sofisticación: los paramentos ortogonales blancos encalados contrastan con el pavimento de losa tradicional de Cadaqués; o la amplia superficie vidriada del estar se protege con persianas enrollables de caña (Fig. 08-09).

En resumen, la casa de Lanfranco Bombelli en Cadaqués da muestra de un ensayo cabal de arquitectura 
doméstica. Una casa-con-arquitecto en el más amplio sentido de la palabra. Su experimentalismo plástico de autor es palpable y se imbrica con una comprensión del programa de la vivienda absolutamente moderno y personal, así como con una sofisticada relectura de los lenguajes constructivos que es sensible con la tradición local. En definitiva, la Casa LBT es una casa compuesta por tres iniciales, pero ideada por un solo arquitecto. La persona que está detrás de ella — su autor y a la vez habitante- es sin duda una de las claves fundamentales con las que comprender esta casa, que es a un tiempo proyecto gráfico y proyecto de arquitectura.

Notas

1 El nombre «LBT» viene de la denominación que recibía el proyecto en los propios planos encontrados en el Arxiu Històric del COAC. Con fecha a seis de junio de 1961, esta última versión parece estar dibujada por el mismo Lanfranco, ya que aparecen sus iniciales en la carátula en el apartado «drawn».

2 Como ya se ha mencionado anteriormente, la familia de Harnden contaba con un total de seis miembros.

3 Sobre la arquitectura de Cadaqués se recomiendan los textos de Anna Noguera Nieto y Manuel Martín Madrid, El Cadaqués de Peter Harnden y Lanfranco Bombelli (Barcelona: Col-legi d'Arquitectes de Catalunya, 2003); Red, "A Cadaqués», Domus 384 (1961): 35-41; Miguel Centellas Soler et al., La vivienda moderna: registro DOCOMOMO ibérico: 1925-1965 (Barcelona: Fundación Caja de Arquitectos / DOCOMOMO Ibérico, 2009); y Federico Correa Ruiz, "Cadaqués», Arquitectura 65 (1964): 32-41.

4 Aunque es la firma Harnden Bombelli la que rubrica el proyecto, el hecho de tratarse de su propia casa, de las sutiles diferencias que presenta respecto al resto, y el hecho significativo de que los planos encontrados en el archivo histórico de Barcelona están rotulados en italiano, hacen suponer que la autoría de esta casa recae de manera más importante sobre la figura de Bombelli.

5 Como Villa Gloria (1959), Casa Staempfli (1960), Casa Mary Calleri (1961) y Casa Bordeoux Groult (1961).

6 Las calles en cuestión son Colomer y Santa Bárbara.

7 Carme Tena Llorens y Ferrán Lorenzo Patiño, «Casa Bombelli», en Noguera y Martín, El Cadaqués, 76-77.

8 Bombelli organizó la exposición «Arte astratta e concreta» que se celebró en Milán del 11 de enero al 9 de febrero de 1947 en colaboración con Max Huber, y siempre con el asesoramiento de Max Bill. Un amplio elenco internacional de primerísima fila expuso sus obras en la muestra, como son Arp, Bill, Kandinsky, Lohse, Munari, Rho, Sottsass, Taeuber-Arp o Vontongerloo. En el catálogo, además, se reunían textos de Kandinsky, Bill, Ettore Sottsass y Vontongerloo (Cf. Jean Arp et al., Arte astratta e concreta. Esposizione organizzata da l'altana, Milano, Palazzo exreale 11 gennaio-9 febbraio 1947 (Milán: Arti grafiche Alfieri e Lacroix, 1947).

9 Noguera y Martín, El Cadaqués, 123.

10 En este sentido, es especialmente revelador el episodio protagonizado por Rogers y Aalto en 1947. En referencia al citado Arte Concreto del que Rogers era firme defensor, el primero — director de la revista «Domus» en aquel momento- le propuso al segundo que escribiera un texto sobre la relación entre la arquitectura y el arte moderno concreto. El resultado dio lugar a un interesantísimo artículo de Aalto, en el que hacía una reflexión acerca de la raíz común abstracta que comparten la arquitectura y las artes, y la capacidad de las nuevas formas para expresar sentimientos con eficacia. Cf. Alvar Aalto, "Arquitectura y Arte Concreto", en Xavier Sust i Fatjó, La humanización de la arquitectura (Barcelona: Tusquets, 1977), 225.

11 También deberían añadirse algunos otros nombres de relieve, como Naum Gabo, Auguste Herbin o el propio Ernesto Nathan Rogers.

12 Jean Arp, Jours effeuillés (Paris: Gallimard, 1966). Texto extraído de Axel Sowa y Serge Demailly, Espace de l'Art Concret. Mouans-Sartoux (Stuttgart/ Londres: Axel Menges, 2005), 7.

13 Además, también resulta interesante leer las reflexiones de Bill en torno a su proyecto no construido para el «Monumento al preso político», donde defiende el arte concreto frente a aquellos que critican que se trate de un arte sin idea, vacío de contenido. Cf. Max Bill et al., La concepción matemática del arte de nuestro tiempo. Max Bill. (Barcelona: Edicions UPC, 2001), 44.

14 El manifiesto, que ocupaba la portada de la revista, explicaba en seis puntos las ideas defendidas por los artistas firmantes del documento: Carlsund, Doesburg, Helion, Tutundjian y Wantz. El posicionamiento de estos artistas se debió a una voluntad de reacción en contra de la pintura abstracta y surrealista dominante: «Nosotros afirmamos: / $1^{\circ} \mathrm{El}$ arte es universal. / $2^{\circ} \mathrm{La}$ obra de arte debe ser totalmente diseñada y formada por la mente antes de su ejecución. No debe recibir las formas dadas de la naturaleza, o de la sensualidad, o del sentimentalismo. Queremos excluir la lírica, el teatro, el simbolismo, etc. / $3^{\circ}$ El cuadro debe ser construido en su totalidad con elementos puramente plásticos, es decir, por planos y los colores. Un elemento pictórico no tiene otro sentido que el sí mismo y en consecuencia el cuadro no tiene otro sentido que el sí mismo. / $4^{\circ}$ La construcción del cuadro, así como sus elementos, debe ser visualmente simple y controlable. / $5^{\circ} \mathrm{La}$ técnica debe ser mecánicamente exacta, anti-impresionista. / 60 Esfuerzo por la claridad absoluta. / (Otto G. Carlsund et al., "Base de la peinture concrète», Art Concret 1 (1930): 1. Texto original en francés, traducción del autor.

15 También conocido, en ocasiones, como abstracción geométrica.

16 El 27 de julio de 1973 se presentaba en un pequeño local de Cadaqués, habilitado para la ocasión, la carpeta Cadaqués Portfolio One. Editada por Lanfranco Bombelli, el objetivo de la iniciativa no era otro que el de recabar los fondos necesarios para adecentar el sepulcro de su recientemente fallecido compañero. En el año 1971, Peter Graham Harnden había fallecido prematuramente, deshaciendo de un modo trágico la más que fructífera relación personal y profesional. Como explica Giralt Miracle, artistas como Richard Paul Lohse, Mary Callery, Max Huber o el propio Lanfranco integraban una lista de doce que no dudaría en colaborar de manera desinteresada en este proyecto en memoria de Peter Harnden. Cf. Daniel Giralt-Miracle, "Cadaqués: portfolio one», Destino 1869 (1973): 31.

17 En relación a la gestalt, habitualmente se consideran como los exponentes de mayor reconocimiento a Max Wertheimer (1923), Wolfgang Köhler (1963), Kurt Koffka (1963), Kurt Lewin y Hartmann (1935).

18 Sobre una reflexión en torno al concepto de multipercepción en el caso de la arquitectura, y con alusión incluida a la mencionada gestalt, se recomienda el texto de Colin Rowe y Robert Slutzky, «Transparency: Literal and Phenomenal (Part 2)», Perspecta 13-14 (1971): $286-301$.

19 En el Arxiu Històric del COAC se conserva un único plano con una versión del proyecto anterior al definitivo. Con fecha del uno de enero de 1961 y con el título "House in Cadaqués. Preliminaries», se presenta un proyecto bastante similar al final en cuanto a la distribución de cada planta, si bien el gran vacío del proyecto se sitúa a la derecha de la misma. El plano mencionado responde a la signatura V89.38.2.

20 Tena y Lorenzo, «Casa Bombelli», 76-77. 
Procedencia de las ilustraciones

Fig. 01-02. Anna Noguera Nieto y Manuel Martín Madrid, El Cadaqués de Peter Harnden y Lanfranco Bombelli. Barcelona: Col•legi d'Arquitectes de Catalunya, 2003.

Fig. 03 y 07. Rafael Zuza Elosegui, fotógrafo (2003).

Fig. 04. Otto G. Carlsund et al., «Base de la peinture concrète», Art Concret 1 (1930): 1.

Fig. 05-06. Daniel Giralt-Miracle, «Cadaqués: portfolio one», Destino, 1869 (1973): 31.

Fig. 08-09. «La casa con la terrazza, nel paese» Domus, 422 (1965): 29-30.

\section{Sobre los autores}

Héctor García-Diego Villarías es arquitecto por la Universidad de Navarra, donde se graduó en 2007 con Premio Extraordinario Fin de Carrera y premio Schindler, además de calificación de Matrícula de Honor en el Proyecto Fin de Carrera. Desde entonces ha desempeńado labores docentes en el departamento de Proyectos, al tiempo que ha coordinado el programa de Actividades Culturales de la escuela. En 2011 recibió una beca de The Getty Trust para una estancia de investigación en The Getty Research Institute de Los Ángeles. Además, ese mismo ańo es becado por la Fundación Bancaja, gracias a lo cual realiza labores de investigación en la Universidad de Columbia en Nueva York como Visiting Scholar. Fruto de estas labores de investigación, obtuvo la calificación de sobresaliente cum laude por su tesis doctoral centrada en las casas de arquitectos extranjeros afincados en Espańa en el tercer cuarto del siglo XX, línea de investigación que en la actualidad continúa desarrollando. En 2013 recibe el Premio Extraordinario de Doctorado.

María Villanueva Fernández es Dr. Arquitecto (2012) con la Tesis Doctoral titulada "Equipar en moderno. Mobiliario de arquitectos españoles". Premio Luis Moya Fin de Carrera (2008), con calificación de Matrícula de Honor en el Proyecto Fin de Carrera. Desde 2008 ha estado vinculada al departamento de Proyectos de la ETSAUN en el área de Expresión Gráfica como profesora en la asignatura de 'Análisis de Formas' (2008-2012) y 'Dibujo Arquitectónico' (2012-2013) y ha sido profesora ayudante del Máster en Diseńo Arquitectónico de la Escuela de Arquitectura de la Universidad de Navarra. Asimismo, desarrolla sus labores docentes en la asignatura 'Creatividad y Estética' (2012-2014) del grado de Ingeniería en Diseño Industrial de la Escuela de Ingenieros de Tecnun y en la asignatura de 'Creatividad' en el Master de ISEM Fashion Business School (2013-2014). Ha realizado estancias de investigación en The Getty Research Institute, Los Ángeles (2011) y en la GSAPP Columbia University, Nueva York (2011), donde ha sido Visiting Scholar.

hgarcia-die@unav.es / mvillanuevf@unav.es 